\title{
Employee grassroot efforts to end structural racism at the US Centers for Disease Control and Prevention
}

To the Editor - On 19 June 2020 (known as Juneteenth), following the civil unrest arising out of the murder of George Floyd and the healthcare inequities highlighted by the COVID-19 (ref. ${ }^{1}$ ) pandemic, a group of concerned staff within the US Centers for Disease Control and Prevention (CDC) initiated a series of discussions to share thoughts and perspectives on these dual pandemics of COVID-19 and racism.

The discussions afforded employees the opportunity to voice their concerns about the CDC's perceived lack of attention to these critical issues. The conversation quickly transitioned from listening sessions to calls for action as questions emerged. Why focus on racism? Why not focus on discrimination, diversity and inclusion? Would focusing on African American employees negate the challenges faced by other marginalized groups? Would the term racism dissuade management's cooperation? How could this group change deeply embedded systems of structural racism that have existed at the CDC for decades, and in our nation since its inception? Will calls for change result in management retaliation ${ }^{2}$ ?

Despite these questions, participants were convinced that inaction was not an option. Employees formed a steering committee, along with sub-committees, to execute delineated goals and objectives. It was agreed that although many groups experience discrimination, the African American experience remains unique, and that addressing racism should remain the primary focus.

Inspired by a letter drafted by US Agency for International Development (USAID) employees urging leadership to address systemic racism within their agency, organizers embarked on a similar course ${ }^{3}$. Organizers crafted an open letter calling for an end to structural racism and outlining seven key actions the agency should take to address these issues - both within the CDC and across the nation. The letter was circulated amongst employees and within the first two weeks more than 1,000 employees signed the letter, which now has more than 1,500 signatories. Of those who have signed, $50 \%$ did so anonymously, with many citing a fear of management retaliation as a reason for doing so.

On 30 June 2020, the letter was shared with the CDC Director and agency

Table 1| The 7 Acts of Change

Declare racism a public health crisis in the United States, and commit resources needed to address its impact on public health

Increase African American representation among senior leadership and diversify the talent pipeline for future leaders

Acknowledge and take immediate, direct action to address the CDC's toxic culture of exclusion and racial discrimination

Dismantle the visible and invisible barriers to career advancement for African American employees

Move from talk to action: implement policy changes that hold our leaders and colleagues accountable for measurable change

Make implicit bias training and cultural sensitivity education mandatory for all staff

Resolve pending Equal Employment Opportunity cases of racial discrimination

leadership ${ }^{4}$. The letter noted that after decades of well-meaning yet underfunded diversity and inclusion efforts, scant progress had been achieved in addressing the very real challenges that African American and other employees of color experience at CDC. These include, but are not limited to, the lack of inclusion in the agency's senior ranks and leadership pipeline programs; a pernicious old boy and girl network that stifles African American talent and blocks opportunities for professional advancement; a pervasive and toxic culture of racial aggressions, bullying and marginalization; a resistance to acknowledging the impact of racism on health; limited accountability, responsibility or leadership regarding these well-documented employee concerns or their relationship to the CDC's overall public health impact in the United States and abroad.

The Director, along with agency leadership, was asked to take immediate, specific and measurable action on the seven actions, known as the '7 Acts of Change' (Table 1).

National public health leaders, many of whom were former CDC staff and had experienced racial discrimination, began to speak out in support of the effort, ultimately forming the Friends of the 7 Acts (known as 'FRIENDS'). This effort of allyship significantly amplified the calls to address systemic injustices within and outside of the CDC's walls. The advocacy of FRIENDS took several forms.

FRIENDS launched an external website $^{5}$ that provides an open forum for the broader public health community to address systemic racism issues at the CDC. FRIENDS also created, and posted on their site, a partner-focused version of the original letter to the CDC leadership. This letter has been signed by 442 individuals external to the agency. In addition, 38 key public health organizations collaborated to speak out in support of the imperatives listed in the 7 Acts of Change letter ${ }^{6}$.

FRIENDS successfully conducted interviews with local and national media outlets and hosted two racism and public health townhall meetings. The first townhall highlighted groups from the CDC, the US National Institutes of Health (NIH) and USAID that were working to address racism at their respective agencies. The second townhall focused on Act 1 Declaring Racism a Public Health Crisis and included presentations from Congresswoman Barbara Lee (DemocratCalifornia and Congressman Tony Cárdenas (Democrat-California), as well as leaders from the American Public Health Association and the American Medical Association. FRIENDS also celebrated Juneteenth with the Inaugural Juneteenth Walk for Change on 19 June 2021 in Atlanta, Georgia, USA.

In August 2020, members of the 7 Acts of Change and the Latino-Hispanic Action Group met with the CDC Director, CDC Principal Deputy Director and the Director of the Office of Minority Health and Health Equity to obtain an update on agency progress toward addressing the actions outlined in the letter. During the meeting, agency leadership communicated a plan 
to elevate and reorganize the Diversity and Inclusion Executive Steering Committee. Beyond this, leadership offered no commitment to fulfill any of the imperatives listed in the letter.

With the appointment of a new CDC Director in January 2021, the 7 Acts of Change members again requested a meeting to discuss ongoing anti-racism efforts at the agency. To date, the new Director has yet to openly acknowledge the 7 Acts of Change or commit to a meeting with the group. However, several other agency leaders have engaged with 7 Acts of Change. In these meetings, concerns have been shared by 7 Acts of Change members that have helped inform the agency's diversity and inclusion agenda.

In April 2021, the nation experienced a milestone in public health with the CDC declaring racism a serious public health threat ${ }^{7}$. This aligned directly with Act 1 of the 7 Acts of Change. Addressing Act 1 is imperative to addressing the well-researched disparities in health between those who self-identify as white and members of all other racial or ethnic groups, with the largest disparities in health status seen between White and Black people ${ }^{8}$.

We acknowledge and celebrate the positive statements and health equity efforts made by the CDC leadership to date. Yet we lament that, dissimilar to the NIH UNITE initiative ${ }^{9}$, the CDC's response shows an aversion to embrace an authentic anti-racism movement. Anti-racism acknowledges the existence of structural racism, identifies where and how it manifests, and seeks to dismantle the levers of power and privilege that perpetuate $\mathrm{it}^{10}$. Initial efforts to support diversity and inclusion by the CDC leadership are also acknowledged. It is understood that this is only the beginning of a long journey to address structural racism in and outside of the agency. The commitment from the 7 Acts of Change members remains strong to pursue meaningful and lasting change at the CDC and beyond.

This includes: continued advocacy for the 7 Acts of Change outlined in the open letter to agency leadership in the summer of 2020; supporting the re-establishment of an official CDC Anti-Racism and Health Workgroup to facilitate research and interventions on racism and health; and serving as an accountability partner to agency leadership in their efforts to address racism and its impact on health in the United States and abroad.

As the 7 Acts of Change members continue with their commitment, we ask the CDC leadership to fully implement each act with fidelity. Change only happens with true buy-in - not just in words, but in deeds.

Christopher S. Parker ${ }^{\bowtie}$, Termika N. Smith, Paul R. Young, Trista Bingham, Lumbé Davis and Eboni Galloway

Atlanta, GA, USA.

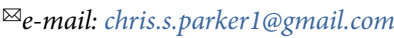

Published online: 7 February 2022

https://doi.org/10.1038/s41591-021-01642-w
References

1. Morbidity and Mortality Weekly Report Vol. 69, No. 33 (CDC, 2020).

Tomaskovic-Devey, D. T., McCann, C. \& Swerzenski, J. D. Government Executive https://www.govexec.com/workforce/ 2021/07/63-workers-who-file-eeoc-discrimination-complaintlose-their-jobs/183849/ (19 July 2021).

3. De Luce, D. \& Williams, A. NBC News https://www.nbcnews. $\mathrm{com} /$ politics/politics-news/usaid-staff-demand-action-agency leaders-over-systemic-racism-n1230316 (11 June 2020).

4. Simmons-Duffin, S. \& Huang, P. NPR https://www.npr.org/ sections/health-shots/2020/07/13/889769017/cdc-employeescall-out-agencys-toxic-culture-of-racial-aggression (13 July 2020).

5. Friends of the 7 Acts of Change https://www.friendsofthe7acts.org (accessed 1 March 2021).

6. San Francisco AIDS Foundation https://www.sfaf.org/collections/ breaking-news/foundation-joins-38-organizations-callingfor-the-cdc-to-declare-racism-a-public-health-crisis/ (4 August 2020).

7. CDC. https://wwwcdc gov/media/releases/2021/s0408-racismhealth.html (8 April 2021)

8. Dovidio, J. F. Soc. Sci. Med. 67, 478-486 (2008)

9. Collins, F. S. et al. Cell 184, 3075-3079 (2021).

10. Jones, C. P. Ethn. Dis. 28, 231-234 (2018).

\section{Acknowledgements}

The authors sincerely thank the following groups for their support, input and collaboration: the Latino-Hispanic

Action Group; the American Indian, Alaska Native and Native Hawaiian Association; the Asian American and Pacific Islander Association; the Disabilities Interest Group; the Friends of the 7 Acts; and the countless supporters of 7 Acts who remain anonymous to protect their

livelihood from backlash and retaliation. The findings and conclusions in this report are those of the authors and do not necessarily represent the official position of the Centers for Disease Control and Prevention or the Agency for Toxic Substances and Disease Registry.

\section{Author contributions}

C.S.P., T.N.S. and P.R.Y. prepared the manuscript, with help from T.B., L.D. and E.G. All authors have reviewed and approve of this submission.

\section{The unique challenges of studying the genetics of diet and nutrition}

To the Editor - Nutrition science has faced a long-standing challenge of establishing causality for nutritional exposures with chronic health outcomes. In the absence of long-term tightly controlled intervention trials, researchers rely on observational studies - where difficulties in measuring food intake and confounding from other behaviors and environmental factors undermine certainty of the evidence. Many would therefore agree that nutrition science is overdue for a renovation.

Epidemiologists increasingly embrace Mendelian randomization (MR), a methodological approach that uses genetic variation associated with modifiable exposures as instrumental variables to infer causal relationships between exposure and outcome $^{1}$. A genotype that determines intake of a specific food or nutrient, randomly assigned at meiosis, could circumvent error and confounding of traditional epidemiologic methods. Could MR therefore be a route to redemption for nutrition science? It seems possible, with monolithic population-based datasets amassing genetic, diet and health outcome data. However, we caution that there are unique challenges to MR for time-varying, compositional and intercorrelated exposures such as diet (Fig. 1).

In MR, a genetic variant serves as a valid instrument for an exposure under certain conditions, including that the genetic variant is associated with the exposure, does not share common causes with the health outcome, and affects the outcome only through its effect on the exposure of interest ${ }^{2}$. It is proposed that if these assumptions hold, then a genetic variant for an exposure acts similarly to randomized treatment allocation in a randomized clinical trial. However, changes in habitual diet over time - sudden or gradual - are one reason nutritional exposures pose unique challenges to the MR framework.

Despite harboring a genetic preference or aversion for a particular food, humans often deviate from their genetic susceptibility. 